\title{
Partial, non-thrombotic, superior sagittal sinus occlusion due to occipital skull tumours
}

\author{
G T Plant, J J Donald, A Jackowski, S J Vinnicombe, B E Kendall
}

\begin{abstract}
Two cases are described in which raised intracranial pressure occurred as a result of superior sagittal sinus (SSS) occlusion by an occipital skull tumour. One was a plasmacytoma, the other a metastatic deposit from a Ewing's sarcoma. The difficulties in diagnosis of this syndrome are illustrated together with the importance and success of appropriate treatment. From the literature it appears possible that these two tumours may be particularly likely to occlude the SSS and the reasons for this are discussed.
\end{abstract}

Superior sagittal sinus (SSS) thrombosis is a well recognised cause of raised intracranial pressure. Less attention has been paid to the fact that midline tumours in the occipital region of the skull can give rise to raised intracranial pressure secondary to non-thrombotic compressive occlusion of dural venous sinuses. The purpose of this article is to report two such cases illustrating the difficulties in making the diagnosis if the possibility is not actively considered. The importance of recognising the syndrome is also clear as both lesions were successfully treated: one case was treated by excision of the tumour and we know of no previous report of a similar case managed surgically. A survey of the literature suggests that the underlying pathologies in these two cases, plasmacytoma and Ewing's sarcoma, may be particularly likely to produce raised intracranial pressure by this mechanism.

Middlesex Hospital, London, UK

Department of

Neurology

G T Plant

Department of

Neurosurgery

A Jackowski

Department of

Radiology

J J Donald

B E Kendall

Department of Medicine

$\mathrm{S} J$ Vinnicombe

Correspondence to

Dr Plant, The National

Hospital for Neurology and

Neurosurgery, Queen

Square, London WC1N 3BG, UK

Received 13 March 1990 and in revised form 20 July 1990

Accepted 1 November 1990 was treated with intermittent chemotherapy for ten years with a good response. The course of the illness was one of recurrent plasmacytomas, often producing pathological fractures, without evidence of bone marrow plasmacytosis. During the course of the disease he developed paraplegia secondary to compression of the spinal cord by a myeloma deposit in the body of T8. This responded to treatment with steroids and radiotherapy.

At the age of 54 he complained of mild headaches and was noted to have bilateral papilloedema. There were no other abnormal signs on neurological examination. Visual acuity was $6 / 6$ bilaterally and the blind spots enlarged on visual field testing. A CT brain scan was carried out but neither enlargement of the ventricular system nor a mass lesion were found and the scan was at first considered to show no abnormality. CSF examination revealed an opening pressure of greater than $40 \mathrm{~cm}$ of CSF. The protein content was $0.26 \mathrm{~g} / \mathrm{L}$ and the glucose $5.5 \mathrm{mmol} / \mathrm{L}$ with no increase in cellularity and no malignant cells seen on cytological examination. He was followed for a further four weeks during which time he began to notice loss of vision. On examination he then showed constriction of visual fields and he was admitted for further investigation. A further CT brain scan showed midline destruction of the occipital bone with an associated soft tissue mass (fig 1A). In retrospect this lesion had been visible, although considerably less obvious, on the first CT scan. A digital subtraction angiogram (DSA) showed in the venous phase partial occlusion of the SSS with an adjacent "tumour blush" (fig 1B).

$\mathrm{He}$ was treated with $20 \mathrm{gy}$ to the whole brain in five fractions with steroid cover. Over two weeks there was further visual loss and his acuity fell to $6 / 18$ bilaterally despite a further therapeutic lumbar puncture. A second DSA showed no improvement in the occlusion of the sinus. It was therefore decided, eight weeks following the diagnosis of raised intracranial pressure, to treat the lesion surgically. An occipital craniotomy was performed and at operation the plasmacytoma was readily peeled off the dura of the compressed SSS, which visibly re-expanded at the end of the procedure. Following surgery there was an immediate symptomatic improvement in visual function. A month later the optic discs were flat and the visual fields were normal, including the size of the blind spots, but the acuity impaired at $6 / 18$ bilaterally. Six months later there had been no further improvement and optic atrophy was noted.

\section{Case two}

A 19 year old warehouseman developed progressive left hip and knee pain over a period of six months. He then experienced severe generalised headaches associated with vomiting. Four days following the onset of the headaches he experienced visual obscurations and intermittent horizontal diplopia. On examination he was afebrile without neck stiffness. Movements of the left hip and knee were 

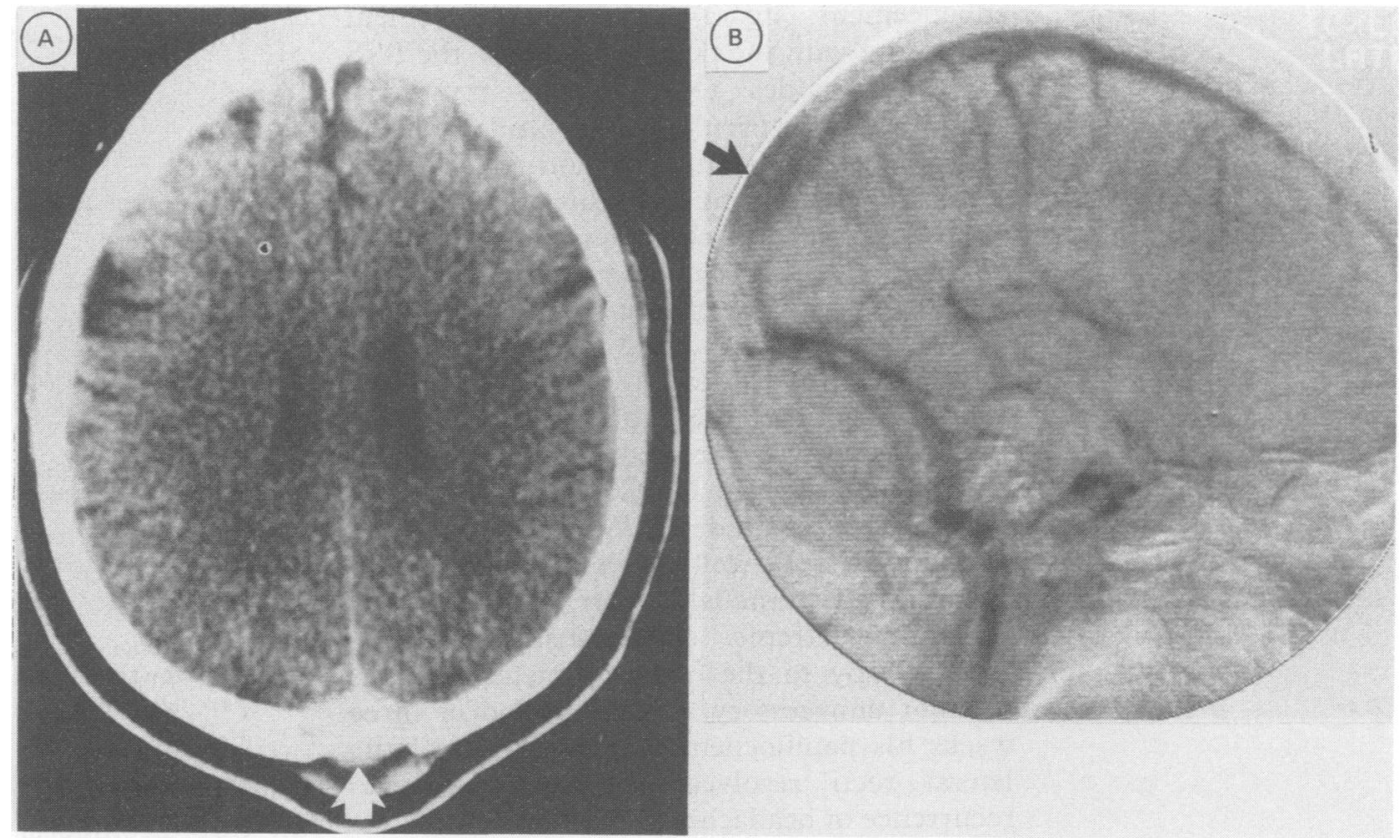

Figure 1 (A) Contrast enhanced CT brain scan (case one) showing a midline occipital skull defect due to a plasmacytoma (arrow).

(B) Venous phase of DSA (case one) showing compression of the terminal portion of the SSS by the plasmacytoma, visible as a "tumour blush" (arrow).

painful. Visual acuity was normal but the blind spots were enlarged on examination of the visual fields. There was bilateral weakness of the lateral recti and, on fundoscopy, bilateral papilloedema with haemorrhages. There were no focal neurological signs in the limbs.

A radiograph of the left femur revealed an extensive permeative lytic lesion involving the mid shaft with a periosteal reaction. A needle biopsy showed histological features typical of Ewing's sarcoma. A chest radiograph was normal. An unenhanced CT scan of the brain showed ventricles of normal size and no evidence of an intracranial mass. A lumbar puncture was carried out. The opening pressure was greater than $40 \mathrm{~cm}$ of CSF, and CSF was withdrawn until the pressure was reduced to $14 \mathrm{~cm}$ of $\mathrm{CSF}$. The CSF protein was $0.12 \mathrm{~g} / \mathrm{L}$, glucose $4.0 \mathrm{mmol} / \mathrm{L}$, there was no increase in cellularity and no malignant cells were seen on cytological examination. Following this procedure his headaches resolved and visual obscurations abated. An intravenous DSA was carried out which showed marked extrinsic compression of the SSS at the level of the internal occipital protuberance (fig 2A). An isotope bone scan of the whole skeleton was later carried out showing increased uptake in the left femur, knee and ankle and at the inion (fig 2B).

Following these investigations the CT scan was repeated. A scan with bone windows confirmed a prominent internal occipital protruberance but there was no evidence of bone destruction. A CT scan with contrast

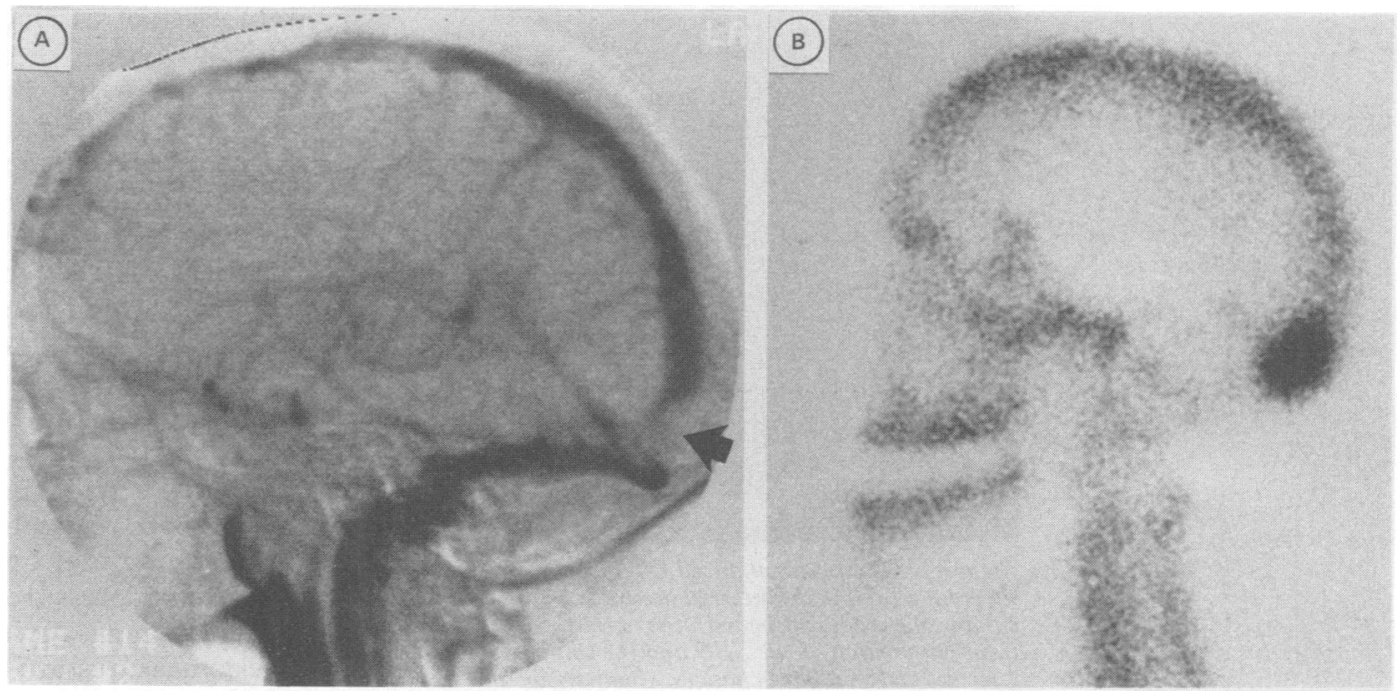

Figure 2 (A) Venous phase of DSA (case two) showing a filling defect in the terminal portion of the SSS which is compressed at this point (arrow).

(B) Isotope bone scan showing high uptake at the inion (case two). 
enhancement (fig 3) showed enhancement coinciding with the position at which the SSS normally divides to form the transverse sinuses (the torcular Herophili). These appearances are similar to normal enhancement of the dural sinuses at this point. In view of the fact that this coincides with the site of obstruction of the sinus on the DSA it was concluded that the enhancement was not of the sinus but an extradural metastatic deposit which had not caused any alteration in the appearance of the inner table of the skull.

The patient was treated with combination chemotherapy on the UKCCSG protocol with IVAD (Ifosfamide, Vincristine and Adriamycin) as well as with concomitant radiotherapy to the left femur. Because of the tumour's extreme chemosensitivity, local radiotherapy to the occipital metastasis was thought unnecessary. Over a period of three weeks his papilloedema and weakness of the lateral recti resolved and there was no recurrence of headaches.

Follow up DSA (fig 4: four weeks following the examination shown in fig $2 \mathrm{~A}$ ) showed a normal appearance of the SSS in the venous phase of the angiogram with no evidence of compression. The CT scan was not repeated.

\section{Discussion}

The most recent review articles which have discussed raised intracranial pressure due to dural sinus occlusion have concentrated on dural sinus thrombosis. ${ }^{12}$ Published cases of SSS occlusion associated with neoplastic disease have usually been examples of thrombosis secondary to tumour infiltration or compression of the sinus. Cases of disseminated carcinoma of the breast with SSS thrombosis are des-

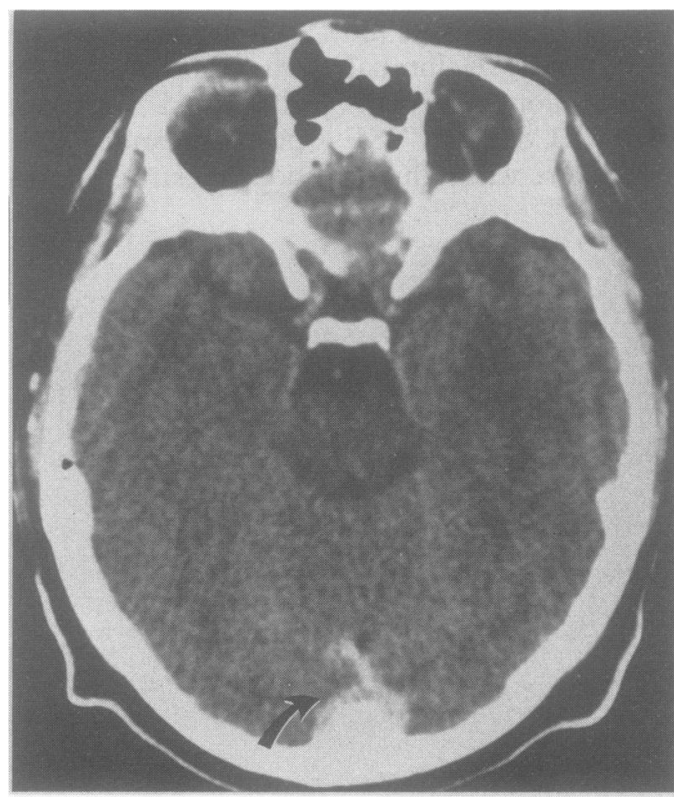

Figure 3 Contrast enhanced CT brain scan (case two) showing a prominent internal occipital protuberance and enhancement coinciding with the normal position of the terminal portion of the SSS and the confluence of sinuses (arrow) without bone changes. Comparison with the DSA (fi $2 A$ ) indicates that the sinus is compressed at this point and the appearances must indicate enhancement of the metastasis.

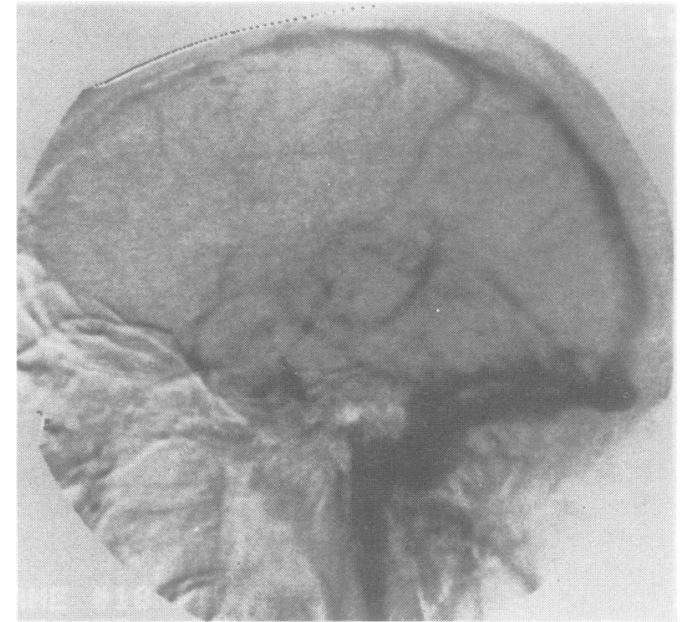

Figure 4 Following treatment of case two with chemotherapy the SSS no longer appears compressed (compare fig $2 A$ ).

cribed by Averback ${ }^{3}$ and reviewed by Sigsbee $e t$ $a l .{ }^{4} \mathrm{SSS}$ thrombosis may also occur as a "nonmetastatic" complication of cancer. ${ }^{4}$ Mones $^{5}$ and, Korenman and Mones, ${ }^{6}$ however, described a series numbering 18 cases of neoplastic disease with evidence of raised intracranial pressure without focal signs and without hydrocephalus or mass lesions. These cases have been incorrectly quoted as examples of SSS compression leading to thrombosis ${ }^{47}$ but in fifteen cases it was shown angiographically or at necropsy that the SSS and torcular were occluded or partially occluded by extradural compression without thrombosis.

This distinction is important because although SSS thrombosis can present without focal signs ${ }^{8}$ often there are, in addition to headache and signs attributable to raised intracranial pressure, clinical features resulting from involvement of cortical veins notably seizures, paresis and other focal signs. ${ }^{9}$ The two cases reported here emphasise the lack of focal signs in non-thrombotic occlusion of the sinus.

It is interesting that of the fifteen patients in the series cited above ${ }^{56}$ four were cases of Ewing's sarcoma with secondary deposits in the skull (four were neuroblastomas and five were cases of carcinoma of the breast). More recently a case of Ewing's tumour with a metastasis to the jugular foramen has been reported with raised intracranial pressure but this resulted from thrombosis of the transverse sinus. ${ }^{7}$ Furthermore, four cases have been described previously ${ }^{10}$ of solitary plasmacytomas of the cranial vault arising in the diploe, destroying both tables of the skull and extending in part extradurally to cause displacement of the venous sinuses. Raised intracranial pressure was present in three of the cases.

As far as intracranial tumours are concerned, it has been recognised for some years that meningiomas may occasionally produce raised intracranial pressure by occluding a venous sinus ${ }^{11}$ and it has recently been emphasised that unless measures are taken to improve venous drainage as well as resect the tumour papilloedema may persist and visual loss continue. ${ }^{12}$ 
The site of compression has been similar in nearly all of the reported cases-the terminal portion of the SSS and the torcular Herophili. Obstruction at this site will produce raised pressure throughout the length of the SSS. Impaired absorption of CSF from the arachnoid granulations will occur and raised intracranial pressure will develop if anastomotic channels between the SSS and elements of the cerebral venous system proximal to the site of obstruction are inadequate. This syndrome will only occur when the tumour produces an extradural tissue mass beneath the inner table of the skull. This is perhaps less likely to occur with some tumours commonly giving rise to bone metastases, such as carcinoma of the bronchus, where expanding lytic lesions in the skull without a soft tissue mass are more usual. The failure to develop adequate drainage of the SSS through anastamotic venous channels may be influenced by individual anatomic variation and also by the rate at which venous obstruction takes place. This rate is presumably much slower when the sinus is compressed by a meningioma and removal of a portion of the SSS may be safely done if it has already been slowly occluded by tumour. ${ }^{1314}$

In a patient with a known neoplasm and raised intracranial pressure it may be considered adequate to exclude ventricular dilatation or an intracranial mass lesion. However, where metastatic disease is a possibility, intravenous contrast enhancement is essential and if no gross structural lesion is found then problem areas should be reviewed including the possible sites of venous sinus obstruction. The cases reported here emphasise that the syndrome of non-thrombotic partial occlusion of the SSS should be actively considered in cases of neoplasm presenting with raised intracranial pressure without focal signs, particularly cases of myeloma in adults and of Ewing's sarcoma and neuroblastoma in children and young adults.

We are grateful to Professor R L Souhami and Dr C J Earl for permission to describe patients under their care and to Professor Souhami for his comments on an earlier draft.

1 Kalbag RM, Woolf AL. Cerebral phlebothrombosis and thrombophlebitis. In: Handbook of clinical neurology. Vol 12. New York: Elsevier, 1972:422-46.

2 Humphrey PRD, Clark CRA, Greenwood RJ. Cerebral venous thrombosis. In: Harrison MJG, Dyken ML, eds. Cerebral vascular disease. London: Butterworths, 1983 309-19.

3 Averback P. Primary cerebral venous thrombosis in young adults: the diverse manifestations of an underrecognised disease. Ann Neurol 1978;3:81-6.

4 Sigsbee B, Deck MDF, Posner JB. Nonmetastatic superior sagittal sinus thrombosis complicating systemic cancer. Neurol (New York) 1979;29:139-46.

5 Mones RJ. disease of the venous sinuses. A report of six cases. Neurol (Minneap) 1965;15:1000-7.

6 Korenman G, Mones RJ. Carcinomatous involvement of the superior sagittal sinus. Trans Am Neurol Assoc 1970; 95:271-2.

7 Graus F, Slatkin NE. Papilledema in the maetastatic jugular formamen syndrome. Arch Neurol 1983;40:816-18.

8 Ray BS, Dunbar H. Thrombosis of the superior sagittal sinus as a cause of pseudotumour cerebri. Trans $\mathrm{Am}$ Neurol Assoc 1950;75:12-17.

9 Dubois J. Les thrombophlébites cérébrales et méningie du post partum. Gynéc et Obstét 1956;55:472-93.

10 Jakubowski J, Kendall BE, Symon L. Primary plasmacytomas of the cranial vault. Acta Neurochirurgica 1980;55:117-34.

11 Marr WG, Chambers JW. Occlusion of the cerebral dural sinuses. Am J Ophthalmol 1966;61:45-49.

12 Repka MX, Miller NR. Papilledema and dural sinus obstruction. J Clin Neuro-ophthalmol 1984;4:247-50.

13 Dandy WE. Removal of longitudinal sinus involved in tumours. Arch Surg 1940;41:244-60

14 Jaeger JR. Ligation and resection of the superior longitudinal sinus. Arch Neurol Psychiat 1942;48:977-87. 